\title{
Are Platelet Distribution and Volume Values Parameters for Meniere's Disease?
}

\author{
Fatih Turan ${ }^{1}\left(\mathbb{D}\right.$, Sena Genç Elden² ${ }^{\mathbb{D}}$, Mahmut Sinan Yılmaz ${ }^{(\mathbb{D}}$, Mahmut Emre Gündogan ${ }^{3}$ (D) \\ Mehmet Güven ${ }^{3}$ (D)
}

\author{
${ }^{1}$ Sakarya University Training and Research Hospital, Department of Otorhinolayngology, Sakarya, Turkey \\ ${ }^{2}$ Pamukova State Hospital, Department of Otorhinolayngology, Sakarya, Turkey \\ ${ }^{3}$ Sakarya University Faculty of Medicine, Department of Otorhinolaryngology, Sakarya, Turkey
}

ORCID ID: F.T. 0000-0002-5389-8243; S.G.E. 0000-0002-3479-1600; M.S.Y. 0000-0002-5323-0059; M.E.G. 0000-0001-9225-8268; M.G. 0000-0002-3665-2428

Citation: Turan F, Genc Elden S, Yilmaz MS, Gundogan ME, Guven M. Are platelet distribution and volume values parameters for meniere's disease?. Tr-ENT 2021;31(4):94-99. https://doi.org/10.26650/Tr-ENT.2021.997578

\begin{abstract}
Objective: To evaluate the relationship between mean platelet volume (MPV), platelet distribution width (PDW), as well as the other parameters of complete blood counts (CBC) and diagnosis and prognosis in Meniere's disease (MD).

Material and Methods: Complete blood count data of 54 MD patients who were followed in our clinic between 2010 and 2018 and age/sexmatched controls were analyzed retrospectively. MPV, PDW, and the other parameters of CBC were compared. Subjects with normal serum glucose, cholesterol, vitamin, and liver and kidney function test levels were included in the study. Subjects with chronic diseases that may affect CBC values were excluded. The Meniere's group was divided into subgroups according to the degree of hearing loss and CBC values were compared within subgroups.

Results: MPV and PDW values were significantly high in the Meniere's group. There was no significant difference in the other parameters of CBC between the MD and control groups. Statistically significant correlation was obtained in MPV and PDW values in the Meniere's group. As the audiometry values increased, MPV and PDW were found to increase.

Conclusion: MPV and PDW, which are markers of vascular pathology, might be potential new serum markers in Meniere's disease.
\end{abstract}

Keywords: Complete Blood Counts; Meniere's Disease, Mean platelet volume, Platelet distribution width, Sensorineural hearing loss

\section{INTRODUCTION}

Meniere's Disease (MD) is defined as an idiopathic syndrome characterized by recurrent episodes of vertigo, hearing loss, fullness in the ear, tinnitus, and endolymphatic hydrops in the inner ear (1). The hearing loss in MD is of the sensorineural type and is initially in fluctuant style, which holds low frequencies $(200-600 \mathrm{~Hz})(2)$. As the course of MD progresses, the hearing level decreases at other frequencies and flat hearing loss occurs (3).

Although many factors are suspected in the etiology of MD, the exact cause is still unclear (4). Endolymphatic hydrops has been the most important finding in studies on the pathophysiology of MD (5). Endolymphatic hydrops is associated with endolymph release, absorption dysfunction, and endolymphatic duct obstruction (6). Factors such as genetic predisposition, autoimmune disease, inflammation, endocrine system abnormalities, viral infections, vascular system abnormalities, allergy, syphilis, leukemia, and trauma are at fault in the hydrops mechanism.

Vascular mechanisms of MD have been attributed to decreased intracerebral arterial pressure, venous obstruction, chronic CSF pressure increase, and chronic hypoxia, which reduce the perfusion rate (7). It has been reported that disruption of the venous absorption mechanism of endolymph as a result of these mechanisms may be associated with vascular pathology $(5,8,9)$. The vascular pathology that disrupts this hemostasis

Corresponding Author: Sena Genç Elden E-mail: ssenagenc@gmail.com

Submitted: $20.09 .2021 \bullet$ Accepted: 09.12.2021 • Published Online: 04.01.2022 
balance in $\mathrm{MD}$ is the presence of venous insufficiency in the paravestibular canalicular (PVC) vein network, which is very important for the inner ear fluid mechanisms (10).

Platelets involved in hemostasis play a role in the formation of thrombosis. Platelets secrete mediators that are important for coagulation, thrombosis, atherosclerosis, and inflammation (11). MPV shows the function and activity of platelets. Volumetrically large platelets are more metabolically and enzymatically active and have greater thrombotic potential (11). Mean platelet volume (MPV) is a marker used in systemic inflammatory conditions and cardiovascular pathologies (12). Platelet distribution width (PDW) represents heterogeneity in platelet morphology. MPV and PDW values are more specific parameters than platelet count in evaluating platelet function (12). MPV and PDW are complete blood count elements that can be performed quickly and cheaply in routine blood tests.

The amount of MPV and PDW is closely related to cardiovascular risk factor such as atherosclerosis, acute syndromes, venous and arterial thrombosis, carotid and peripheral artery disease, or thromboembolism (13-15). In the study by Ulu et al., MPV value was found to be significantly higher in patients with sudden hearing loss and it was associated with vascular pathologies (16). Likewise, in the study by Sagit et al., MPV and PDW values were found to be significantly higher in patients with sudden hearing loss (17).

In the present study, we aimed to evaluate MPV and PDW, as well as the other parameters of complete blood counts (CBC), supporting the hypothesis of vascular pathology in $\mathrm{MH}$. To the best of our knowledge, this is the first study to evaluate $C B C$ parameters in MD.

\section{MATERIALS AND METHODS}

Ethics committee approval was obtained from the Local Ethics Committee (Ethics Committee No:71522473/050.01.04/162). The study was carried out by retrospectively examining the records of patients diagnosed with MD who applied to the ENT clinic of Sakarya University Training and Research Hospital.

Fifty-four patients who presented with peripheral vertigo attack and were diagnosed with MD after detailed anamnesis, physical examination and tests were included in the study. Routine blood test results and audiometry values of these patients during the attack period and at the 3rd month after the attack were evaluated. Patients were evaluated with respect to $C B C$, glucose, cholesterol, liver function tests, renal function tests, and vitamin B9 and B12. Audiometric and vestibular tests (VHIT, VNG) were performed to differentiate peripheral and central vertigo. It was requested that patients who had not been diagnosed with neurological diseases be evaluated for them. At the end of this process, patients diagnosed with MD were included in the study. The control group was selected from the patients who applied to the otorhinolaryngology outpatient clinic for routine checkups. Patients in the control group who were found to have pathology (such as vertigo, nasal septum deviation, nasal polyposis, sensory hearing loss, obstructive sleep apnea syndrome, malignancy) in the detailed otolaryngology examination were excluded from the study. People with any chronic disease were not included in either group so that the $C B C$ value was not affected.

Neutrophil, lymphocyte, neutrophil/lymphocyte ratio (NLR), platelet/lymphocyte ratio (PLR) MPV, and PDW values were compared. Glucose, cholesterol, liver function tests, renal function tests, and vitamin values were evaluated in both groups, in order to avoid any chronic disease that might affect $\mathrm{CBC}$ values.

The affected ear was identified in the Meniere's group. Meniere's patients were divided into subgroups according to the degree of hearing loss on the affected ear side during the non-attack periods and during the attack periods. Hearing losses were found to be $0-25 \mathrm{~dB}$ normal hearing, 26-40dB mild sensorineural hearing loss ( $\mathrm{SNHL}$ ), 41-70dB medium SNHL, 7190dB advanced SNHL, and 91dB + profound SNHL, according to the pure sound averages of $500,1000,2000$, and $4000 \mathrm{~Hz}$.

\section{Statistical Analysis}

Statistical analysis was performed using SPSS (Statistical Package for Social Sciences) 22.0 program. Hematological data were analyzed by the Shapiro-Wilk test for normal distribution in each group and subgroups. Descriptive results of the data with normal distribution according to normality distribution were stated as mean $\pm S D$, and non-normal distribution was defined as median [IR]. The normal distribution data of the groups were compared with the independent-samples t-test and the non-normal distribution data were compared with the Mann-Whitney $U$ test. The normal distribution of CBC data of subgroups according to hearing loss in the Meniere's group were compared with the one way ANOVA test, and the nonnormal distribution data were compared with the Kruskal Wallis test. MPV and PDW results which were statistically significant for these groups were evaluated by linear regression test. Results were evaluated at $95 \%$ confidence interval and $p<0.05$ significance level.

\section{RESULTS}

The mean age was $48.15 \pm 11.91$ years and consisted of 26 (48.1\%) women and 28 (51.9\%) men in the Meniere's group. The mean age was $45.05 \pm 7.72$ years and consisted of 25 (46.3\%) women and 29 (53.7\%) men in the control group. There was no significant difference between Meniere's and control groups in terms of age, sex, glucose, cholesterol, liver function tests, renal function tests, and vitamin values $(p \geq 0.05)$ (Table 1$)$.

While there was no significant difference in neutrophil, lymphocyte, platelet, NLR, and PLR values between the Meniere's group and the control group in CBC ( $p \geq 0.05)$, a statistically significant difference was found in MPV and PDW values $(p<0.05)$ (Table 2$)$ (Figure 1, Figure 2).

In the Meniere's group, 22 (40.7\%) right and 32 (59.3\%) left ears were affected. Meniere's patients during the attack period of the affected ears had 12 (22.2\%) normal, 13 (24.1\%) mild 
Table 1: Glucose, cholesterol, renal and liver function tests and B12, folic acid mean values between control and Meniere's groups.

\begin{tabular}{lccc}
\hline Variable & Meniere's group & Control group & $p$ value \\
\hline Glucose $(\mathrm{mg} / \mathrm{d})$ & $100[18.7]$ & $99.5[17]$ & 0.411 \\
Total cholesterol (mg/d) & $198.7 \pm 26.1$ & $199.3 \pm 44.8$ & 0.217 \\
HDL-cholesterol (mg/d) & $49.9 \pm 11.4$ & $45.7 \pm 9.9$ & 0.879 \\
LDL-cholesterol (mg/d) & $124.5 \pm 26.8$ & $129.4 \pm 35.7$ & 0.758 \\
AST (U/L) & $22.5[7]$ & $18.0[7]$ & 0.462 \\
ALT (U/L) & $22.5[13]$ & $21.0[15]$ & 0.809 \\
Serum urea (mg/dL) & $29.2 \pm 7.2$ & $28.6 \pm 8.4$ & 0.374 \\
Creatinine $(\mathrm{mg} / \mathrm{dL})$ & $0.7[0.16]$ & $0.7[0.15]$ & 0.279 \\
Vitamin B12 $(\mathbf{p g} / \mathrm{mL})$ & $357[124.7]$ & $344[181.7]$ & 0.815 \\
Folate $(\mathrm{ng} / \mathrm{mL})$ & $7.0 \pm 2.3$ & $6.46 \pm 2.2$ & 0.816 \\
\hline
\end{tabular}

Continuous variables were presented as mean \pm standard deviation if normal distribution, and median [interquartile range] if not normal distribution. ALT: Alanine Aminotransferase, AST: Aspartate Aminotransferase, HDL: High-Density Lipoprotein, LDL: Low-Density Lipoprotein

Table 2: Mean values of neutrophils, lymphocytes, platelets, NLR, PLR, MPV, PDW between Meniere's and control groups.

\begin{tabular}{|c|c|c|c|}
\hline Variable & Meniere's group & Control group & $\mathrm{p}$ value \\
\hline Neutrophil count $\left(10^{9} / \mathrm{L}\right)$ & $3.9[1.7]$ & $3.9[2.3]$ & 0.982 \\
\hline Lymphocyte count $\left(10^{9} / \mathrm{L}\right)$ & $2.1[1.2]$ & $2.5[0.9]$ & 0.490 \\
\hline Platelet count $\left(10^{3} / \mathrm{mm}^{3}\right)$ & $253.6 \pm 53.5$ & $261.0 \pm 61.4$ & 0.537 \\
\hline MPV (fl) & $7.98[1.81]$ & $7.53[1.18]$ & $0.023^{*}$ \\
\hline PDW (fl) & $18.2 \pm 1.1$ & $17.5 \pm 0.6$ & $0.001^{*}$ \\
\hline NLR & $1.68[1.12]$ & $1.60[1.11]$ & 0.372 \\
\hline PLR & $106.50[48.50]$ & $106.32[53.79]$ & 0.949 \\
\hline
\end{tabular}

Continuous variables were presented as mean \pm standard deviation if normal distribution, and median [interquartile range] if not normal distribution. *A statistically significant difference was obtained between MPV and PDW values.

MPV: Mean Platelet Volume, PDW: Platelet Distribution Width, NLR: Neutrophil/Lymphocyte Ratio, PLR: Platelet/Lymphocyte Ratio

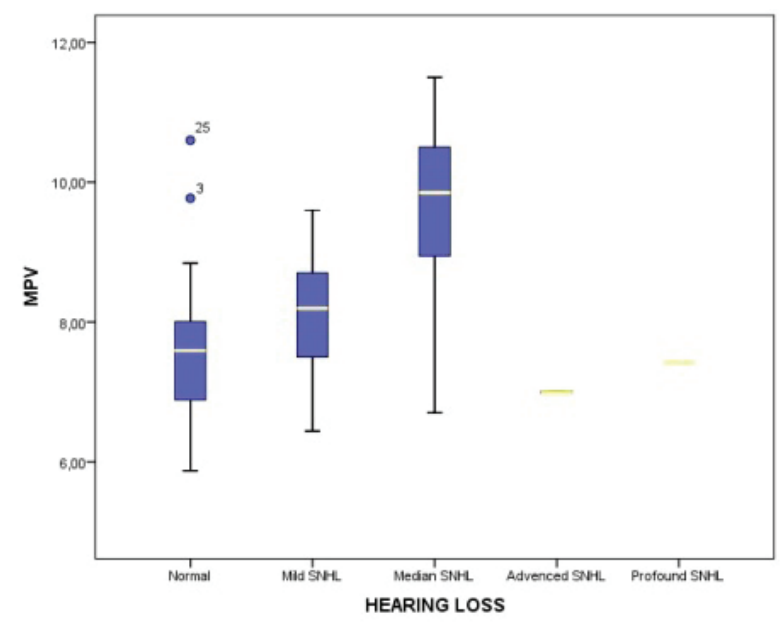

Figure 1: Distribution of MPV value between groups.

SNHL, 25 (46.3\%) medium SNHL, 2 (3.7\%) advanced SNHL, and $2(3.7 \%)$ profound SNHL according to the pure sound averages of $500,1000,2000$, and $4000 \mathrm{~Hz}$. There were no significant difference in $C B C$ values according to the degree of hearing loss ( $p \geq 0.05$ ) (Table 3 ).

During the nonattack period, 20 (37.1\%) of Meniere's patients had normal hearing, 19 (35.1\%) mild SNHL, 12 (22.2\%) medium SNHL, and 2 (3.7\%) advanced SNHL, and 1 (1.9\%) patient had

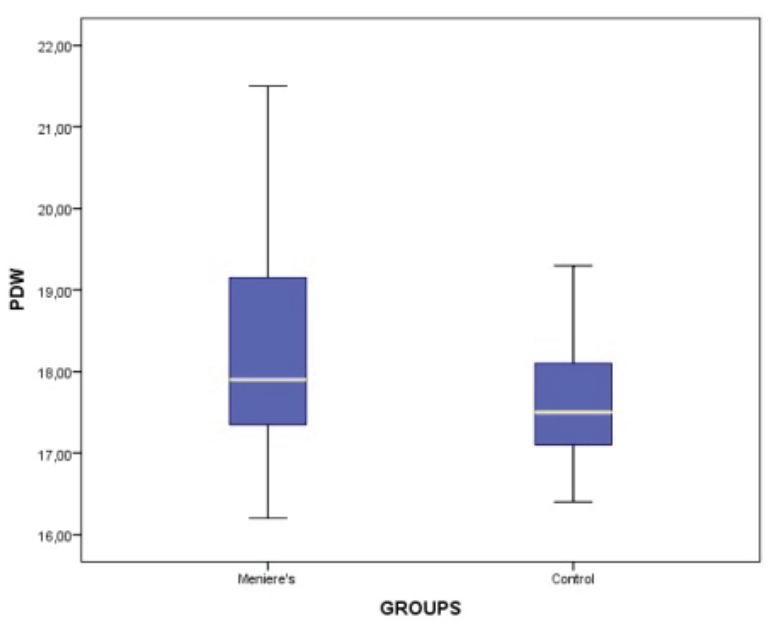

Figure 2: Distribution of PDW value between groups.

profound SNHL according to the pure sound averages of 500 , 1000,2000 , and $4000 \mathrm{~Hz}$. A significant correlation was found between MPV and PDW levels and the degree of hearing loss during the non-attack period $(p<0.05)$. When verification was performed by regression analysis, the difference in MPV and PDW values was significant ( $p<0.05$ ) (Figure 3 ) (Figure 4). There was no significant difference in the other $\mathrm{CBC}$ values between groups ( $p \geq 0.05$ ) (Table 4 ). 
Table 3: Meniere's group, mean values of neutrophils, lymphocytes, platelets, NLR, PLR, MPV, PDW according to hearing loss during attack period.

\begin{tabular}{|c|c|c|c|c|c|c|}
\hline Variable & $\begin{array}{l}\text { Normal } \\
(n=12)\end{array}$ & $\begin{array}{l}\text { Mild SNHL } \\
\quad(n=13)\end{array}$ & $\begin{array}{l}\text { Medium SNHL } \\
(n=25)\end{array}$ & $\begin{array}{l}\text { Advanced SNHL } \\
\qquad(n=2)\end{array}$ & $\begin{array}{l}\text { Profound SNHL } \\
\qquad(n=2)\end{array}$ & $p$ value \\
\hline Neutrophil count (109/L) & $4.0 \pm 1.0$ & $3.9 \pm 1.2$ & $4.8 \pm 2.6$ & $3.9 \pm 0.6$ & $4.8 \pm 3.4$ & 0.749 \\
\hline Platelet count $\left(10^{3} / \mathrm{mm}^{3}\right)$ & $263 \pm 62$ & $269 \pm 63$ & $245 \pm 48$ & $244 \pm 41$ & $219 \pm 10$ & 0.656 \\
\hline MPV (fl) & $7.6 \pm 1.3$ & $8.3 \pm 0.8$ & $8.7 \pm 1.5$ & $6.9 \pm 0.2$ & $7.1 \pm 0.4$ & 0.112 \\
\hline PDW (fl) & $17.5[1.3]$ & $17.5[2.1]$ & $18.9[1.7]$ & $18.8[-]$ & $16.9[-]$ & 0.078 \\
\hline PLR & $121 \pm 58$ & $109 \pm 28$ & $114 \pm 34$ & $134 \pm 49$ & $84 \pm 25$ & 0.395 \\
\hline
\end{tabular}

Continuous variables were presented as mean \pm standard deviation if normal distribution, and median [interquartile range] if not normal distribution. PDW: Platelet Distribution Width, NLR: Neutrophil/Lymphocyte Ratio, PLR: Platelet/Lymphocyte Ratio

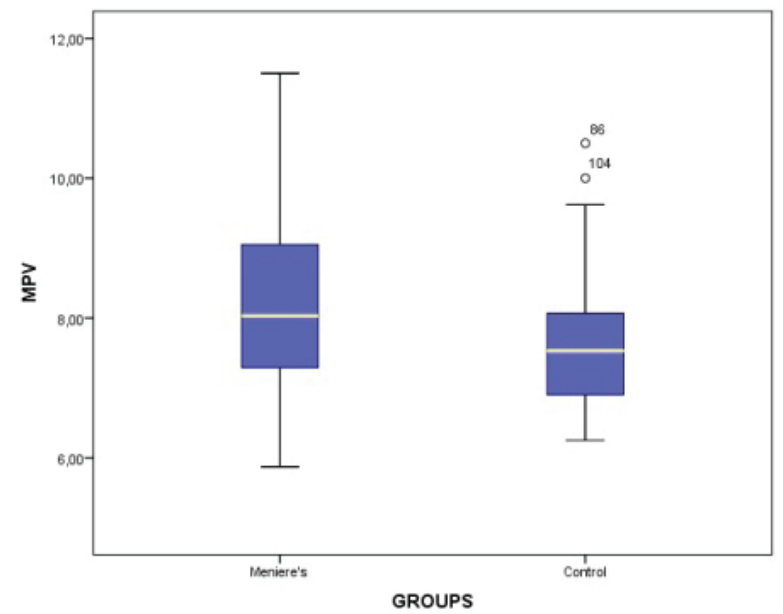

Figure 3: MPV distribution according to the degree of hearing loss in non-attack period.

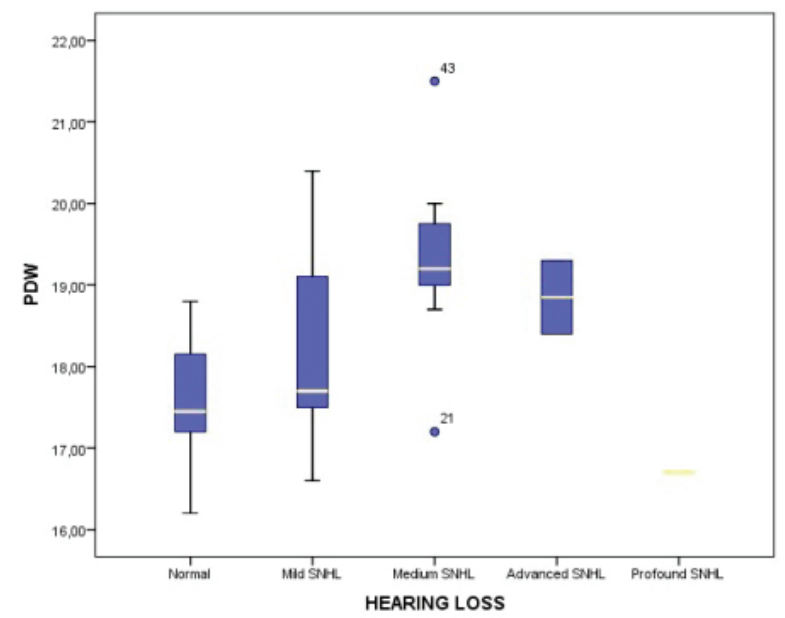

Figure 4: PDW distribution according to the degree of hearing loss in non-attack period.

Table 4: Meniere's group, mean values of neutrophils, lymphocytes, platelets, NLR, PLR, MPV, PDW according to hearing loss during nonattack period.

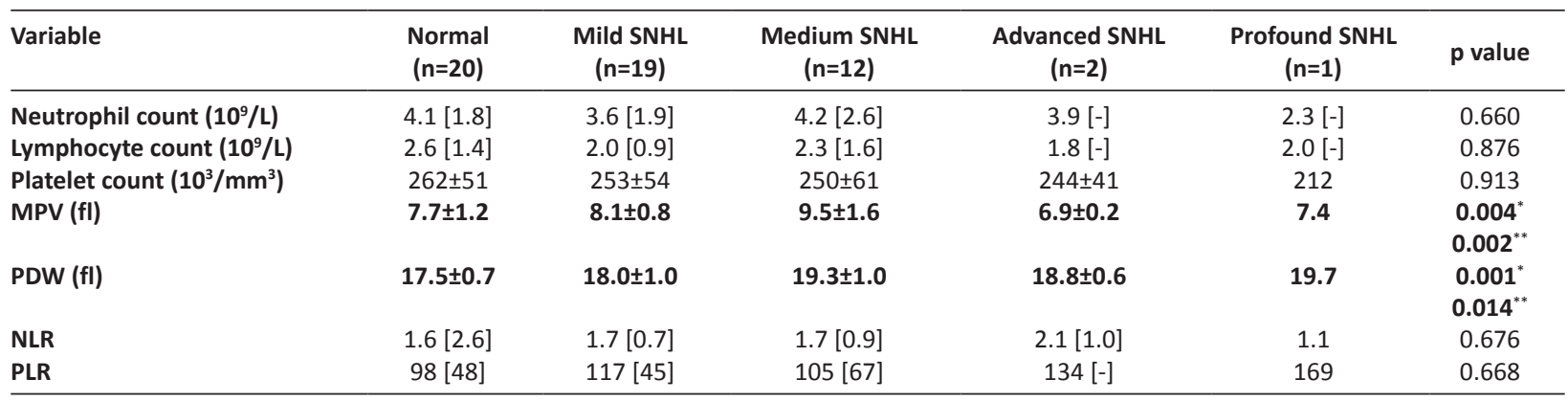

Continuous variables were presented as mean \pm standard deviation if normal distribution, and median [interquartile range] if not normal distribution. MPV: Mean Platelet Volume, PDW: Platelet Distribution Width, NLR: Neutrophil/Lymphocyte Ratio, PLR: Platelet/Lymphocyte Ratio *There is a statistically significant difference between MPV and PDW values.

**In the regression analysis, there was a significant difference in MPV and PDW values.

\section{DISCUSSION}

Hematologic data were used to evaluate the vascular pathologies that are important in the pathogenesis of MD in the present study. To the best of our knowledge, this is the first study to evaluate complete blood count parameters in MD. MPV and PDW values were significantly high in the Meniere group. In addition, there was a significant correlation between hearing loss and MPV and PDW values in the Meniere group.

The male to female ratio in MD was found to be $1.3 / 1$ in several studies, but in the present study, the ratio was $1 / 1 .(4,14)$. Although the course of MD can be highly variable, it usually occurs in the fourth to seventh decade, with episodic vertigo 
or sensorineural hearing loss affecting low frequencies. In the present study, the mean age was found to be $48.15 \pm 11.917$ in accordance with the literature.

Increased PVC venous pressure leads to inadequate drainage of vestibular organs and is one of the important mechanisms in the pathophysiology of MD (10). Friberg et al., in their study, emphasized the classical triad of MD, vertigo, tinnitus, and hearing loss as the only symptom of radiologically detected thrombosis of the sigmoid sinus and jugular bulbus (18). In another study, it was shown that cerebral atherosclerosis, transient ischemic attack, MD, and equilibrium disorders can be seen together with the common etiology of vascular pathology and the underlying mechanism of this status was thought to be due to possible episodic labyrinth ischemia (19).

MPV is a measure of platelet size. Platelets play an important role in initiating atherosclerosis and thrombogenesis (3). Large platelets contain more dense alpha granules, express more adhesion receptors, and have higher thrombotic activity (20). Therefore, MPV is a marker of platelet activation. MPV value is a more specific parameter than platelet count in the evaluation of platelet function (3). MPV is a marker used in systemic inflammatory conditions and cardiovascular pathologies (12). There is a close association between MPV and cardiovascular risk factors such as impaired fasting glucose levels, diabetes mellitus, hypertension, hypercholesterolemia, obesity, and metabolic syndrome $(12,20)$.

PDW represents heterogeneity in platelet morphology and is clinically associated with platelet activation, such as MPV (21). Increased MPV and PDW reflect increased platelet activation or an increased number of large, hyper aggregated platelets and are considered an independent risk factor for coronary and peripheral arterial disease $(13,14,22)$. MPV and PDW values in the present study support vascular pathology. Endler et al. reported that increased MPV and PDW values were independent risk factors for acute myocardial infarction, and other studies have also shown that they are associated with increased mortality and recurrent vascular events after myocardial infarction (23-25).

In a study which examined the $\mathrm{CBC}$ results of patients presenting with peripheral vertigo, only NLR values were found to be significant among the other CBC values (26). We were unable to duplicate this result as we did not find a significant difference in NLR values. The reason for this might be due to the low number of patients with MD in our study. In another study comparing $C B C$ values of peripheral vertigo patients with vestibular neuritis, significant results were obtained in NLR, PLR and MPV values (27). The significant results in NLR and PLR values were attributed to inflammatory pathology and the absence of significant results in the present study might indicate that MD is not primarily an inflammatory pathology.

A significant result in MPV value in the present study suggests the important role of the vascular process in MD. Several other researchers examined $\mathrm{CBC}$ results in patients with sudden $\mathrm{SNHL}$ and tinnitus. In these studies, a significant increase was found in NLR rates, but no significant change was found in MPV values and they associated their results with inflammatory pathology $(28,29)$. In the study in which patients with sudden hearing loss were grouped according to their audiometry grade, it was found that the MPV value was significantly higher in patients with total deafness and in whom all frequencies were affected compared to the control group (30). In the present study, MPV and PDW values showed a correlation with hearing loss in Meniere's patients, suggesting that there may be a correlation between the severity of MD and MPV and PDW values.

\section{CONCLUSION}

MPV and PDW values indicate vascular pathologies that are important in the pathogenesis of MD. Our study suggests that MPV and PDW may be potential new markers in evaluating MD, and there may be a significant relationship between MPV and PDW values and disease course and severity. Since the number of patients with advanced and profound SNHL is limited in our study, further studies with MD with advanced hearing loss are required to support our data.

Ethics Committee Approval: Ethics committee approval was obtained from the Local Ethics Committee (Ethics Committee No:71522473/050.01.04/162).

Informed Consent: Written informed consent was obtained.

Peer-Review: Externally peer-reviewed.

Author Contributions: Conception/Design of Study- F.T., S.G.E., M.E.G.; Data Acquisition- F.T., S.G.E., M.E.G.; Data Analysis/Interpretation- F.T., S.G.E., M.S.Y.; Drafting Manuscript- F.T., S.G.E., M.G.; Critical Revision of Manuscript- F.T., S.G.E., M.G., M.S.Y.; Final Approval and AccountabilityF.T., S.G.E., M.S.Y., M.E.G., M.G.

Conflict of Interest: Authors declared no conflict of interest.

Financial Disclosure: Authors declared no financial support.

\section{REFERENCES}

1. Ciccone MM, Scicchitano P, Gesualdo M, Cortese F, Zito A, Manca $\mathrm{F}$, et al. Idiopathic sudden sensorineural hearing loss and Meniere syndrome: The role of cerebral venous drainage. Clin Otolaryngol 2018;43(1):230-9.

2. Havia M, Kentala E. Progression of symptoms of dizziness in Meniere's disease. Arch Otolaryngol Head Neck Surg 2004;130(4):431-435.

3. Mateijsen DJ, Van Hengel PW, Van Huffelen WM, Wit HP, Albers FW. Puretone and speech audiometry in patients with Meniere's disease. Clin Otolaryngol Allied Sci 2001;26(5):379-387.

4. Liu $\mathrm{Y}, \mathrm{Xu} \mathrm{H}$. The intimate relationship between vestibular migraine and Meniere disease: a review of pathogenesis and presentation. Behav Neurol 2016;2016:3182735.

5. Paparella MM, Djalilian HR. Etiology, pathophysiology of symptoms, and pathogenesis of Meniere's disease. Otolaryngol Clin North Am 2002;35(3):529-45.

6. Paparella MM. Pathogenesis and pathophysiology of Meniere's disease. Acta Otolaryngol 1991;485:26-35. 
7. Foster CA, Breeze RE. The Meniere attack: an ischemia/ reperfusion disorder of inner ear sensory tissues. Med Hypotheses 2013;81(6):1108-15.

8. Bruno A, Napolitano M, Califano L, Attanasio G, Giugliano V, Cavazzuti PP6, et al. The Prevalence of Chronic Cerebrospinal Venous Insufficiency in Meniere Disease: 24-Month Follow-up after Angioplasty. J Vasc Interv Radiol 2017;28(3):388-91.

9. Mancini F, Catalani M, Carru M, Monti B. History of Meniere's disease and its clinical presentation. Otolaryngol Clin North Am 2002;35(3):565-80.

10. Gussen R. Vascular mechanisms in Meniere's disease. Theoretical considerations. Arch Otolaryngol 1982;108(9):544-9.

11. Karli R, Alacam H, Unal R, Kucuk H, Aksoy A, Ayhan E. Mean platelet volume: is it a predictive parameter in the diagnosis of sudden sensorineural hearing loss? Indian J Otolaryngol Head Neck Surg 2013;65(4):350-3.

12. Demirkol S, Balta S, Unlu M, Yuksel UC, Celik T, Arslan Z, et al. Evaluation of the mean platelet volume in patients with cardiac syndrome X. Clinics (Sao Paulo) 2012;67(9):1019-22.

13. Mayer FJ, Hoke M, Schillinger M, Minar E, Arbesú I, Koppensteiner $\mathrm{R}$, et al. Mean platelet volume predicts outcome in patients with asymptomatic carotid artery disease. Eur J Clin Invest 2014;44(1):22-8.

14. Berger JS, Eraso LH, Xie D, Sha D, Mohler ER 3rd. Mean platelet volume and prevalence of peripheral artery disease, the National Health and Nutrition Examination Survey, 1999-2004. Atherosclerosis 2010;213(2):586-91.

15. Braekkan SK, Mathiesen EB, Njølstad I, Wilsgaard T, Størmer $J$, Hansen JB Mean platelet volume is a risk factor for venous thromboembolism: the Troms $\varnothing$ Study, Troms $\varnothing$, Norway. J Thromb Haemost 2010;8(1):157-62.

16. Ulu S, Ulu MS, Ahsen A, Yucedag F, Aycicek A, Celik S. Increased levels of mean platelet volume: a possible relationship with idiopathic sudden hearing loss. Eur Arch Otorhinolaryngol 2013;270(11):2875-8.

17. Sagit M, Kavugudurmaz M, Guler S, Somdas MA. Impact of mean platelet volume on the occurrence and severity of sudden sensorineural hearing loss. J Laryngol Otol 2013;127(10):972-6.

18. Friberg $\mathrm{U}$, Rask-Andersen $\mathrm{H}$. Vascular occlusion in the endolymphatic sac in Meniere's disease. Ann Otol Rhinol Laryngol 2002;111(3 Pt 1):237-45.

19. Hood NA. Diseases of the central nervous system. Cerebral atherosclerosis, transient ischaemic attacks, Menière's disease, and disorders of balance. $\mathrm{Br}$ Med J 197515;4(5993):398-400.
20. Sansanayudh N, Numthavaj P, Muntham D, Yamwong S, McEvoy $M$, Attia J, et al. Prognostic effect of mean platelet volume in patients with coronary artery disease. A systematic review and meta-analysis. Thromb Haemost 2015;114(6):1299-309.

21. Yang SW, Cho SH, Kwon HS, Sohn IS, Hwang HS. Significance of the platelet distribution width as a severity marker for the development of preeclampsia. Eur J Obstet Gynecol Reprod Biol 2014;175:107-11.

22. Zheng YG, Yang T, Xiong CM, He JG, Liu ZH, Gu Q, et al. Platelet distribution width and mean platelet volume in idiopathic pulmonary arterial hypertension. Heart Lung Circ 2015;24(6):56672.

23. Endler $G$, Klimesch A, Sunder-Plassmann H, Schillinger M, Exner $\mathrm{M}$, Mannhalter $\mathrm{C}$, et al. Mean platelet volume is an independent risk factor for myocardial infarction but not for coronary artery disease. Br J Haematol 2002;117(2):399-404.

24. Huczek Z, Kochman J, Filipiak KJ, Horszczaruk GJ, Grabowski $M$, Piatkowski $R$, et al. Mean platelet volume on admission predicts impaired reperfusion and long-term mortality in acute myocardial infarction treated with primary percutaneous coronary intervention. J Am Coll Cardiol 2005;46(2):284-90.

25. Rechciński T, Jasińska A, Foryś J, Krzemińska-Pakuła M, Wierzbowska-Drabik K, Plewka M, et al. Prognostic value of platelet indices after acute myocardial infarction treated with primary percutaneous coronary intervention. Cardiol J 2013;20(5):491-8.

26. Ozbay I, Kahraman C, Balikci HH, Kucur C, Kahraman NK, Ozkaya DP, et al. Neutrophil-to-lymphocyte ratio in patients with peripheral vertigo: a prospective controlled clinical study. Am J Otolaryngol 2014;35(6):699-702.

27. Şahin MI, Kokoğlu K, Gulmez E. Mean platelet volume, neutrophiland platelet to lymphocyte ratios are elevated in vestibular neuritis. J Clin Neurosci 2019;67:134-38.

28. Gunes A, Karali E, Ural A, Ruzgar F, Bayatkara T. The relationship of high-frequency distortion product otoacoustic emission (DPOAE) values with hematological parameters in tinnitus patients. Eur Arch Otorhinolaryngol 2019;276(11):3013-9.

29. Lee JS, Hong SK, Kim DH, Lee JH, Lee HJ, Park B, et al. The neutrophilto-lymphocyte ratio in children with sudden sensorineural hearing loss: a retrospective study. Acta Otolaryngol 2017;137(1):35-8.

30. Sun Y, Guo Y, Wang H, Chen Z, Wu Y, Shi, H, et al. Differences in platelet-related parameters among patients with audiographically distinct sudden sensorineural hearing loss: A retrospective study. Medicine (Baltimore) 2017;96(36):e7877. 